Pacific Journal of Mathematic 


\title{
POLYNOMIAL COVERING SPACES AND HOMOMORPHISMS INTO THE BRAID GROUPS
}

\author{
VAGN LUNDSGAARD HANSEN
}

\begin{abstract}
A polynomial covering space is the zero set for a parametrized family of complex polynomials. Such covering spaces arise naturally in several contexts in the study of holomorphic mappings. There are fruitful connections between polynomial covering spaces and certain homomorphisms into the Artin braid groups. We shall establish these connections and use them to obtain an algebraic classification of the polynomial covering spaces.
\end{abstract}

1. Introduction. Covering spaces defined by parametrized families of complex polynomials arise naturally in several contexts in the study of holomorphic mappings, e.g., in connection with the Weierstrass preparation theorem. A study of the topology of such covering spaces within a proper topological framework was suggested in [6] by the introduction of polynomial covering spaces. Roughly speaking, an $n$-fold polynomial covering space over a connected, topological space $X$ is the zero set for a continuously varying family of simple, normed, complex polynomials of degree $n$ parametrized by $X$. It is well known that an $n$-fold covering space over $X$ is classified by a conjugacy class of homomorphisms of the fundamental group of $X$ into the symmetric group on $n$ letters. The purpose of this paper is to examine the relationship between $n$-fold polynomial covering spaces over $X$ and homomorphisms of the fundamental group of $X$ into the Artin braid group on $n$ strings. What we are looking for are necessary and sufficient conditions, for a finite covering space over $X$ to be equivalent to a polynomial covering space over $X$, and for two polynomial covering spaces over $X$ to be equivalent as coverings.

In [4] and later papers, see e.g., the references cited in [5], Gorin and Lin also study parametrized families of simple, normed complex polynomials, but not from the point of view of examining the topological types of the associated covering spaces. The purpose of Gorin and Lin is to investigate, when a continuous family of polynomials admits a continuous factorization in polynomials of degree 1 over the parameter space. The existence of such a factorization is equivalent to the associated polynomial covering space being trivial. Of other papers somehow related to the work in this paper, we note in particular the papers by Arnol'd [1] and Zjuzin and $\operatorname{Lin}[9]$. 
Now a short description of the contents of the paper. The link from $n$-fold polynomial covering spaces over $X$ to homomorphisms of the fundamental group of $X$ into the braid group on $n$ strings is established in $\S 2$ via the introduction of root maps and their induced homomorphisms. In $\S 3$ we describe certain characteristic homomorphisms for an $n$-fold polynomial covering space over $X$, with Lemma 3.1 as the key result. Section 4 contains a geometric criterion for a polynomial covering space to be trivial, stated as Theorem 4.1. This is the point, where contact is made with the work of Gorin and Lin [4]. We comment on that in Remark 4.2. Section 4 closes with Example 4.3, which shows among others that a nontrivial homomorphism into the braid group on $n$ strings can determine a trivial $n$-fold covering space. The main results in the final $\S 5$ are Theorem 5.1, where we give an algebraic criterion for a covering space to be equivalent to a polynomial covering space, and Theorem 5.7, where we give a solution to the problem, when two homomorphisms of the fundamental group of $X$ into the braid group on $n$ strings determine equivalent $n$-fold polynomial covering spaces over $X$. Theorem 5.7 provides thereby an algebraic classification of the $n$-fold polynomial covering spaces over $X$.

The author is indepted to the University of Warwick for hospitality in the month of July 1977, when part of this work was done, and to the referee for pointing out to him the work of Gorin and Lin.

2. The link from polynomial covering spaces to homomorphisms into the braid groups. Throughout this paper $X$ denotes a connected topological space with the homotopy type of a $C W$-complex and with a nondegenerate base point $x_{0} \in X$. The fundamental group of $X$ with base point $x_{0} \in X$ will be denoted by $\pi_{1}(X)$.

First we recall the basic definitions from [6]. A simple Weierstrass polynomial $P(x, z)$ over $X$ is a polynomial function $P: X \times C \rightarrow C$ of the form

$$
P(x, z)=z^{n}+\sum_{i=1}^{n} a_{i}(x) z^{n-i},
$$

where $a_{1}, \cdots, a_{n}: X \rightarrow C$ are complex valued, continuous functions, such that for any fixed $x \in X, P(x, z)$ has no multiple complex roots. Associated with $P(x, z)$ there is an $n$-fold covering map $\pi: E \rightarrow X$ defined by the diagram,

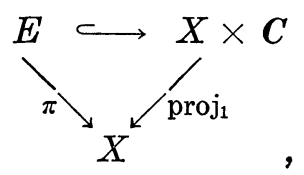


where $E=\{(x, z) \in X \times C \mid P(x, z)=0\}$ and proj $_{1}$ is projection onto the first factor. $\pi: E \rightarrow X$ is called the $n$-fold polynomial covering map, and $E$ the $n$-fold polynomial covering space over $X$, associated with $P(x, z)$.

The continuous functions $\alpha_{i}: X \rightarrow C, i=1, \cdots, n$, define a (continuous) map

$$
a=\left(a_{1}, \cdots, a_{n}\right): X \longrightarrow B^{n}=C^{n} \backslash \Delta
$$

into the complement of the discriminant set $\Delta \cong C^{n}$ in complex $n$ space $C^{n} . \quad a: X \rightarrow B^{n}$ is called the coefficient map for the polynomial covering map $\pi: E \rightarrow X$ associated with $P(x, z)$. We know from ([6], $\S 3)$ that $\pi: E \rightarrow X$ is equivalent to the pull-back of a canonical $n$ fold polynomial covering map $\pi^{n}: E^{n} \rightarrow B^{n}$ along $a$. Therefore $n$-fold polynomial covering spaces over $X$, which have homotopic coefficient maps, are equivalent as coverings.

In order to put polynomial covering spaces into connection with conjugacy classes of homomorphisms from the fundamental group of $X$ into the braid groups we need some preparation.

Let $\Sigma_{n}$ denote the symmetric group on $n$ letters. The action of $\Sigma_{n}$ on the space of complex $n$-tuples $C^{n}$ by permutation of coordinates induced a free $\Sigma_{n}$-action on

$$
F_{n}(\boldsymbol{C})=\left\{\left(z_{1}, \cdots, z_{n}\right) \in \boldsymbol{C}^{n} \mid z_{i} \neq z_{j}, \text { all } i \neq j\right\}
$$

with the configuration space $C_{n}(\boldsymbol{C})$ for an (unordered) set of $n$ points in the plane $C$ as orbit space. The canonical projection map

$$
p_{n}: F_{n}(\boldsymbol{C}) \longrightarrow C_{n}(\boldsymbol{C})
$$

defines therefore a principal $\Sigma_{n}$-bundle, in particular an $n$ !-fold covering map. It is well known, see e.g., ([6], §4), that there is a homeomorphism

$$
\Phi: C_{n}(C) \longrightarrow B^{n},
$$

which to the element $\operatorname{cls}\left(\alpha_{1}, \cdots, \alpha_{n}\right) \in C_{n}(\boldsymbol{C})$ associates the $n$-tuple $\left(a_{1}, \cdots, a_{n}\right) \in B^{n}$ defined by

$$
z^{n}+\sum_{i=1}^{n} a_{i} z^{n-i}=\left(z-\alpha_{1}\right) \cdots\left(z-\alpha_{n}\right) .
$$

By a theorem of Fadell and Neuwirth [2] we know that $C_{n}(\boldsymbol{C})$, and hence also $B^{n}$, is an Eilenberg-MacLane space of type $(B(n), 1)$, where $B(n)$ denotes the Artin braid group on $n$ strings. Take $c_{0}=$ $(1, \cdots, n) \in F_{n}(C), \bar{c}_{0}=p_{n}\left(c_{0}\right) \in C_{n}(C)$ and $b_{0}=\Phi\left(c_{0}\right) \in B^{n}$ as base points in $F_{n}(C), C_{n}(C)$ and $B^{n}$, respectively. Then the group of braids on $n$ strings can be defined as the fundamental group $B(n)=\pi_{1}\left(C_{n}(\boldsymbol{C}), \bar{c}_{0}\right)$. 
The group $H(n)=\pi_{1}\left(F_{n}(\boldsymbol{C}), c_{0}\right)$ is called the group of colored braids on $n$ strings. For later reference we note that the homotopy sequence for the covering map $p_{n}: F_{n}(\boldsymbol{C}) \rightarrow C_{n}(\boldsymbol{C})$ reduces to the following short exact sequence, which we will call the braid group sequence,

$$
1 \longrightarrow H(n) \stackrel{\rho_{n}}{\longrightarrow} B(n) \stackrel{\tau_{n}}{\longrightarrow} \Sigma_{n} \longrightarrow 1 \text {. }
$$

$\rho_{n}$ is induced by the projection map $p_{n}$ and $\tau_{n}$ is a boundary operator, called the permutation homomorphism.

Let now $\pi: E \rightarrow X$ be an $n$-fold polynomial covering map with coefficient map $a: X \rightarrow B^{n}$. Using the homeomorphism $\Phi: C_{n}(C) \rightarrow B^{n}$ we can then define a unique map $\alpha: X \rightarrow C_{n}(C)$ such that the following diagram is commutative,

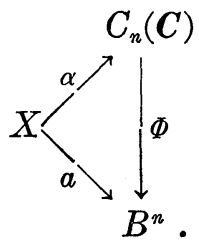

For obvious reasons $\alpha: X \rightarrow C_{n}(\boldsymbol{C})$ is called the root map for the polynomial covering map $\pi: E \rightarrow X$. By definition of $\Phi$, the coefficient map $\alpha$ and the root map $\alpha$ are related by the formula

$$
z^{n}+\sum_{i=1}^{n} a_{i}(x) z^{n-i}=\prod_{z_{x} \in \alpha(x)}\left(z-z_{x}\right)
$$

for all $x \in X$.

The root map $\alpha: X \rightarrow C_{n}(\boldsymbol{C})$ induces a homomorphism

$$
\alpha_{*}: \pi_{1}\left(X, x_{0}\right) \longrightarrow \pi_{1}\left(C_{n}(C), \alpha\left(x_{0}\right)\right)
$$

between fundamental groups. Since $x_{0} \in X$ is a nondegenerate base point and $C_{n}(\boldsymbol{C})$ is connected, this homomorphism determines a unique conjugacy class of homomorphisms of $\pi_{1}(X)$ into $B(n)$. Any one of the homomorphisms in this conjugacy class will also be denoted by

$$
\alpha_{*}: \pi_{1}(X) \longrightarrow B(n) \text {. }
$$

Since $C_{n}(C)$ is an Eilenberg-MacLane space of type $(B(n), 1)$ and $X$ has the homotopy type of a $C W$-complex it is well known, see e.g., Spanier ([7], Theorem 11, p. 428), that there is a bijective correspondence between the free homotopy classes of maps of $X$ into $C_{n}(\boldsymbol{C})$ and the conjugacy classes of homomorphisms of $\pi_{1}(X)$ into $B(n)$ defined by mapping the free homotopy class of $\alpha: X \rightarrow C_{n}(C)$ 
into the conjugacy class of $\alpha_{*}: \pi_{1}(X) \rightarrow B(n)$. The homeomorphism $\Phi: C_{n}(\boldsymbol{C}) \rightarrow B^{n}$ induces a bijective correspondence $\Phi_{*}$ from the free homotopy classes of maps of $X$ into $C_{n}(C)$ to those of $X$ into $B^{n}$. Finally, we get a surjective map by mapping the free homotopy class of $a: X \rightarrow B^{n}$ into the equivalence class of the $n$-fold polynomial covering map $\pi_{a}: E_{a} \rightarrow X$ with coefficient map $a$.

The above remarks are summarized in the following diagram

Conjugacy class of $\alpha_{*}: \pi_{1}(X) \longrightarrow B(n)$

Free homotopy class of $\alpha: X \longrightarrow C_{n}(C)$

Free homotopy class of $a: X \longrightarrow B^{n}$
Hom $\left(\pi_{1}(X), B(n)\right)^{\text {conj. }}$

$\uparrow$ bijective correspondence

$\left[X, C_{n}(C)\right]$

$\Phi_{*} \downarrow$ bijective correspondence

$\left[X, B^{n}\right] \underset{\text { surjective }}{\underset{\operatorname{map}}{\longrightarrow}} P C_{n}(X)$
Equivalence class of $\pi_{a}: E_{a} \longrightarrow X$.

In this diagram Hom $\left(\pi_{1}(X), B(n)\right)^{\text {eonj. }}$ denotes the set of conjugacy classes of homomorphisms of $\pi_{1}(X)$ into $B(n), P C_{n}(X)$ denotes the set of equivalence classes of $n$-fold polynomial covering spaces over $X$, and $[X, Y]$ denotes the set of free homotopy classes of maps of $X$ into $Y$ for an arbitrary space $Y$.

Using the diagram we can freely switch between presenting an equivalence class of $n$-fold polynomial covering spaces over $X$ by a free homotopy class of maps of $X$ into $B^{n}$, or a free homotopy class of maps of $X$ into $C_{n}(\boldsymbol{C})$, or a conjugacy class of homomorphisms of $\pi_{1}(X)$ into $B(n)$.

As we shall see in $\S 4$, the surjective map $\left[X, B^{n}\right] \rightarrow P C_{n}(X)$ is not in general injective.

3. Characteristic homomorphisms for polynomial covering maps. Consider an arbitrary $n$-fold covering map $\pi: E \rightarrow X$. For any ordering of the $n$ points in the fibre over $x_{0} \in X, \pi^{-1}\left(x_{0}\right)$, path lifting induces in an obvious way a homomorphism $\chi(\pi): \pi_{1}(X) \rightarrow \Sigma_{n}$. If we change base point in $X$ or change the order of the points in the fibre over the base point, this changes $\chi(\pi)$ by an inner automorphism in $\Sigma_{n}$. Hence $\chi(\pi)$ is well defined by $\pi$ up to conjugacy. The conjugacy class of $\chi(\pi)$ is exactly the characteristic class of the $n$-fold covering map $\pi$ as defined in Steenrod ([8], 13.5, p. 61). Any choice of a homomorphism $\chi(\pi)$ as above will be called a characteristic homomorphism for $\pi$. It is well known that two $n$-fold covering maps $\pi_{i}: E_{i} \rightarrow X, i=1,2$, are equivalent if and only if $\chi\left(\pi_{1}\right)$, $\chi\left(\pi_{2}\right): \pi_{1}(X) \rightarrow \Sigma_{n}$ are conjugate. See e.g., Steenrod ([8], 13.7, p. 62).

For polynomial covering maps we can give an alternative, and 
in this connection more useful, description of the characteristic homomorphisms.

Let $\pi: E \rightarrow X$ be an $n$-fold polynomial covering map with root map $\alpha: X \rightarrow C_{n}(C)$. As explained in $\S 2, \alpha$ induces a conjugacy class of homomorphisms $\alpha_{*}: \pi_{1}(X) \rightarrow B(n)$. Composing a homomorphism $\alpha_{*}$ in this conjugacy class with the permutation homomorphism $\tau_{n}: B(n) \rightarrow \Sigma_{n}$ from the braid group sequence, we get a homomorphism $\tau_{n} \circ \alpha_{*}: \pi_{1}(X) \rightarrow \Sigma_{n}$. With notation as above we have

LEMMA 3.1. The homomorphism $\tau_{n} \circ \alpha_{*}: \pi_{1}(X) \rightarrow \Sigma_{n}$ is a characteristic homomorphism for the n-fold polynomial covering map $\pi: E \rightarrow X$, i.e., $\chi(\pi)=\tau_{n} \circ \alpha_{*}$.

Proof. Since it is only the conjugacy class of $\alpha_{*}$ that matters, we can assume w.l.o.g. that $\alpha$ is base point preserving, i.e., $\alpha:\left(X, x_{0}\right) \rightarrow\left(C_{n}(C), \bar{c}_{0}\right)$.

Let then $f:[0,1] \rightarrow X$ with $f(0)=f(1)=x_{0}$ be a representative for the element $[f] \in \pi_{1}(X)$, and let $(\alpha \circ f)^{\prime}:[0,1] \rightarrow F_{n}(C)$ be the unique lifting of $\alpha \circ f:[0,1] \rightarrow C_{n}(\boldsymbol{C})$ over $p_{n}: F_{n}(\boldsymbol{C}) \rightarrow C_{n}(\boldsymbol{C})$ with initial value $(\alpha \circ f)^{\prime}(0)=c_{0} \in F_{n}(C)$. Then $(\alpha \circ f)^{\prime}(1)$ defines a permutation $\sigma_{f} \in \Sigma_{n}$ of the $n$ coordinates in $c_{0}=(1, \cdots, n)$. By the construction of $\tau_{n}$ as a boundary operator in the homotopy sequence for $p_{n}$, it is clear that $\tau_{n} \circ \alpha_{*}([f])=\sigma_{f}$.

If we identify the circle $S^{1}$ with the space obtained by identifying end points in $[0,1]$, then $f:[0,1] \rightarrow X$ induces a map $\bar{f}: S^{1} \rightarrow X$, since $f(0)=f(1)=x_{0}$. Let $\bar{f}^{*}(\pi): \bar{f}^{*}(E) \rightarrow S^{1}$ denote the $n$-fold polynomial covering map defined as the pull-back of $\pi$ along $\bar{f}$. From the definition of the characteristic homomorphism $\chi(\pi)$ by path lifting, it follows easily that $\chi(\pi)([f])$ is the clutching permutation, see $([6], \S 8)$, for $\bar{f}^{*}(\pi)$. On the other hand it is clear that the clutching permutation for $\bar{f}^{*}(\pi)$ is $\sigma_{f} \in \Sigma_{n}$. Hence $\chi([f])=\sigma_{f}=\tau_{n}$ 。 $\alpha_{*}([f])$. Since $[f] \in \pi_{1}(X)$ was arbitrary this proves Lemma 3.1.

4. A criterion for a polynomial covering map to be trivial. It is intuitively clear that an $n$-fold polynomial covering map $\pi: E \rightarrow X$ is equivalent to the trivial $n$-fold covering map if and only if we globally can order the set of roots in the underlying simple Weierstrass polynomial in a continuous fashion. This is made precise in the following

THEOREM 4.1. Let $\pi: E \rightarrow X$ be an $n$-fold polynomial covering map with root map $\alpha: X \rightarrow C_{n}(C)$. Then $\pi: E \rightarrow X$ is a trivial covering map if and only if the root map $\alpha$ admits a (continuous) lifting $\alpha^{\prime}: X \rightarrow F_{n}(\boldsymbol{C})$ over $p_{n}: F_{n}(\boldsymbol{C}) \rightarrow C_{n}(\boldsymbol{C})$, 


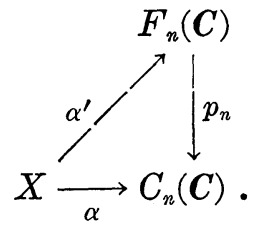

Proof. Assume first, that $\pi: E \rightarrow X$ is a trivial $n$-fold polynomial covering map and let $h: X \times\{1, \cdots, n\} \rightarrow E$ be a homeomorphism commuting with projections onto $X$, which defines an equivalence of $\pi$ with the trivial $n$-fold covering map. Since $E$ is a polynomial covering space, there is an inclusion map $E \hookrightarrow X \times C$ commuting with projections onto $X$. Let $h_{c}: X \times\{1, \cdots, n\} \rightarrow C$ denote the coordinate map for $h$ into $C$, when we consider $h$ as a map into $X \times C$ via this inclusion. Obviously, the root map $\alpha: X \rightarrow C_{n}(C)$ is the map, which to $x \in X$ associates the configuration $\alpha(x) \in C_{n}(C)$ defined by the set of coordinates in $C$ for the $n$ points in $\pi^{-1}(x) \subset$ $X \times C$. The map $\alpha^{\prime}: X \rightarrow F_{n}(C)$, which to $x \in X$ associates the ordered $n$-tuple $\alpha^{\prime}(x)=\left(h_{c}(x, 1), \cdots, h_{c}(x, n)\right) \in F_{n}(C)$ is then clearly a lifting of $\alpha$ as required, i.e., $\alpha=p_{n} \circ \alpha^{\prime}$.

Assume next, that the root map $\alpha: X \rightarrow C_{n}(\boldsymbol{C})$ can be lifted to a map $\alpha^{\prime}=\left(\alpha_{1}, \cdots, \alpha_{n}\right): X \rightarrow F_{n}(\boldsymbol{C})$ over $p_{n}$. The map $h: X \times$ $\{1, \cdots, n\} \rightarrow X \times C$, defined by $h(x, i)=\left(x, \alpha_{i}(x)\right)$ for $x \in X$ and $i=1, \cdots, n$, is then an embedding commuting with projections onto $X$, which maps $X \times\{1, \cdots, n\}$ homeomorphically onto $E$. This proves that $\pi: E \rightarrow X$ is trivial, and hence Theorem 4.1 is proved.

REMARK 4.2. In [4], Gorin and Lin study complete solvability of polynomial equations of the type, which we in this paper call simple Weierstrass polynomials. Complete solvability of a simple Weierstrass polynomial with root map $\alpha: X \rightarrow C_{n}(C)$ is equivalent to the existence of a lifting $\alpha^{\prime}: X \rightarrow F_{n}(C)$ as in Theorem 4.1. See the section preceeding ([4], Lemma 1.1). The results in [4] can therefore immediately be translated into results on triviality of polynomial covering maps.

The author is much indepted to Karsten Grove for coversations on the following

Example 4.3. Consider $S^{1}$ as the space of complex numbers of modulus 1 .

For the simple Weierstrass polynomial $P(x, z)=z^{2}-x^{2}$ over $S^{1}$, the coefficient map $a=\left(a_{1}, a_{2}\right): S^{1} \rightarrow B^{2}=C^{2} \backslash \Delta$ is given by $a(x)=$ $\left(0,-x^{2}\right)$ and the root map $\alpha: S^{1} \rightarrow C_{2}(C)$ is given by $\alpha(x)=\operatorname{cls}(x,-x)$, for $x \in S^{1}$. 
The root map $\alpha: S^{1} \rightarrow C_{2}(C)$ clearly admits a lifting $\alpha^{\prime}: S^{1} \rightarrow F_{2}(C)$ over $p_{2}: F_{2}(\boldsymbol{C}) \rightarrow C_{2}(\boldsymbol{C})$, namely $\alpha^{\prime}(x)=(x,-x)$ for $x \in S^{1}$. Hence the 2-fold polynomial covering map $\pi: E \rightarrow S^{1}$ associated with $P(x, z)$ is trivial by Theorem 4.1. This is also easy to prove directly.

We shall now prove that the coefficient map $a: S^{1} \rightarrow B^{2}$, or equivalently, the root map $\alpha: S^{1} \rightarrow C_{2}(C)$, is not freely homotopic to a constant map. This will show that the surjective map $\left[X, B^{n}\right] \rightarrow$ $P C_{n}(X)$ constructed in $\S 2$ is not in general injective, in particular not for $X=S^{1}$ and $n=2$.

First observe that $F_{2}(C)=\left\{\left(z_{1}, z_{2}\right) \in C^{2} \mid z_{1} \neq z_{2}\right\}$. Hence there is a homeomorphism $F_{2}(\boldsymbol{C}) \rightarrow \boldsymbol{C} \times(\boldsymbol{C} \backslash\{0\})$, which maps $\left(z_{1}, z_{2}\right) \in F_{2}(\boldsymbol{C})$ into $\left(\zeta_{1}, \zeta_{2}\right)=\left(z_{1}+z_{2}, z_{1}-z_{2}\right) \in \boldsymbol{C} \times(\boldsymbol{C} \backslash\{0\})$. Under this homeomorphism, the lifting of the root map $\alpha^{\prime}: S^{1} \rightarrow F_{2}(C), x \mapsto(x,-x)$, obviously corresponds to the map $\tilde{\alpha}^{\prime}: S^{1} \rightarrow C \times(C \backslash\{0\}), x \mapsto(0,2 x)$.

There is an obvious homotopy equivalence $C \times(C \backslash\{0\}) \simeq S^{1}$, defined in the second argument by radial projection. Since $S^{1}$ is a simple space, we can work with based homotopy classes in stead of free homotopy classes of maps.

Using the above remarks it follows that there is a sequence of isomorphisms

$$
\pi_{1}\left(F_{2}(C)\right) \simeq \pi_{1}(C \times(C \backslash\{0\})) \simeq \pi_{1}\left(S^{1}\right) \simeq Z
$$

to the integers $Z$, and that $\alpha^{\prime}: S^{1} \rightarrow F_{2}(C)$ through these isomorphisms corresponds to $1 \in Z$. Hence $\alpha^{\prime}: S^{1} \rightarrow F_{2}(C)$ is not freely homotopic to a constant map. Since $p_{2}: F_{2}(\boldsymbol{C}) \rightarrow C_{2}(\boldsymbol{C})$ is a covering map, this implies that the root map $\alpha: S^{1} \rightarrow C_{2}(C)$ is not freely homotopic to a constant map. This finishes Example 4.3.

5. An algebraic classification of the polynomial covering maps. In the main results in this final section we provide necessary and sufficient conditions, for a homomorphism of $\pi_{1}(X)$ into $\Sigma_{n}$ to represent the equivalence class of an $n$-fold polynomial covering space over $X$, and for two homomorphisms of $\pi_{1}(X)$ into $B(n)$ to define equivalent polynomial covering spaces over $X$.

Most of our investigations are related to the spaces and maps, which will be defined subsequently, in the following commutative diagram,

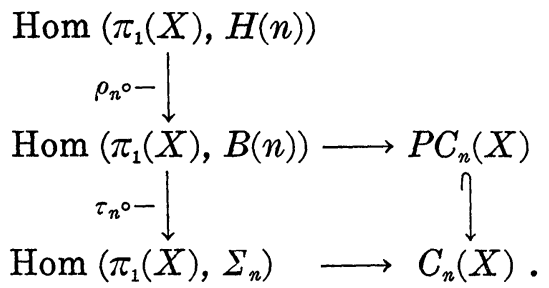


$C_{n}(X)$ and $P C_{n}(X)$ denote respectively the set of equivalence classes of $n$-fold covering spaces over $X$ and $n$-fold polynomial covering spaces over $X$. There is an inclusion map $P C_{n}(X) \hookrightarrow C_{n}(X)$.

For any pair of groups $G$ and $H$, we denote by $\operatorname{Hom}(G, H)$ the set of homomorphisms of $G$ into $H$. The maps $\rho_{n} \circ-$ and $\tau_{n} \circ-$ in the diagram are induced by composition of homomorphisms from the homomorphisms in the braid group sequence

$$
1 \longrightarrow H(n) \stackrel{\rho_{n}}{\longrightarrow} B(n) \stackrel{\tau_{n}}{\longrightarrow} \Sigma_{n} \longrightarrow 1 \text {. }
$$

Finally, we explain now the horizontal maps in the diagram. The map

$$
\operatorname{Hom}\left(\pi_{1}(X), \Sigma_{n}\right) \longrightarrow C_{n}(X)
$$

is the surjective map, which to the homomorphism $\varphi: \pi_{1}(X) \rightarrow \Sigma_{n}$ associates the equivalence class of the $n$-fold covering map $\pi: E \rightarrow X$ having $\varphi$ as a characteristic homomorphism. The covering map $\pi$ can be constructed as an associate bundle to the universal covering space over $X$ viewed as a principal $\pi_{1}(X)$-bundle. Compare also Steenrod ([8], 13.8, p. 64). From the classification theorem for covering maps we know, that two homomorphisms $\varphi, \psi \in$ Hom $\left(\pi_{1}(X), \Sigma_{n}\right)$ determine the same equivalence class of $n$-fold covering spaces over $X$ if and only if they are conjugate. In particular we note, that the constant homomorphism of $\pi_{1}(X)$ into the identity permutation in $\Sigma_{n}$ is the unique representative for the equivalence class of the trivial $n$-fold covering space over $X$.

The map

$$
\operatorname{Hom}\left(\pi_{1}(X), B(n)\right) \longrightarrow P C_{n}(X)
$$

is the surjective map, which to the homomorphism $\varphi: \pi_{1}(X) \rightarrow B(n)$ associates the equivalence class of the $n$-fold polynomial covering map $\pi: E \rightarrow X$ having $\chi(\pi)=\tau_{n} \circ \rho$ as a characteristic homomorphism. That such a map exists follows by the constructions in $\S 2$ combined with Lemma 3.1.

This finishes the definitions of the spaces and maps in the above diagram.

First we make use of the diagram to prove

THEOREM 5.1. The equivalence class of an n-fold covering map $\pi: E \rightarrow X$ represented by the homomorphism $\varphi: \pi_{1}(X) \rightarrow \Sigma_{n}$ contains a polynomial covering map if and only if there exists a homomorphism $\varphi^{\prime}: \pi_{1}(X) \rightarrow B(n)$ such that $\varphi=\tau_{n} \circ \varphi^{\prime}$.

Proof. An $n$-fold polynomial covering space over $X$ is re- 
presented by a homomorphism $\psi: \pi_{1}(X) \rightarrow B(n)$. The homomorphisms $\varphi, \tau_{n} \circ \psi^{\prime} \in \operatorname{Hom}\left(\pi_{1}(X), \Sigma_{n}\right)$ represent equivalent coverings if and only if they are conjugate. Since $\tau_{n}$ is an epimorphism, it follows then easily, that $\varphi$ and $\tau_{n} \circ \psi$ represent equivalent coverings if and only if there exists a homomorphism $\varphi^{\prime}: \pi_{1}(X) \rightarrow B(n)$, conjugate to $\psi: \pi_{1}(X) \rightarrow B(n)$, such that $\varphi=\tau_{n} \circ \varphi^{\prime}$. Theorem 5.1 follows now immediately using the main diagram.

COROLlaRY 5.2. In order that the space $X$ has the property that all n-fold covering spaces over $X$ are equivalent to polynomial covering spaces, it is necessary and sufficient that

$$
\tau_{n} \circ-: \operatorname{Hom}\left(\pi_{1}(X), B(n)\right) \longrightarrow \operatorname{Hom}\left(\pi_{1}(X), \Sigma_{n}\right)
$$

is surjective.

EXAMPLE 5.3. Suppose that $\pi_{1}(X)$ is a free nonabelian group. Then the condition in Corollary 5.2 is clearly satisfied. This is the case, since given a homomorphism $\varphi: \pi_{1}(X) \rightarrow \Sigma_{n}$, we can just lift the images in $\Sigma_{n}$ of a set of generators for $\pi_{1}(X)$ and then extend to a homomorphism $\varphi^{\prime}: \pi_{1}(X) \rightarrow B(n)$. We can lift the images of the generators, since $\tau_{n}: B(n) \rightarrow \Sigma_{n}$ is an epimorphism. For a space $X$ with a free nonabelian fundamental group, any finite covering space over $X$ is therefore equivalent to a polynomial covering space. Specific examples of such spaces $X$ are provided e.g., by punctured surfaces.

Next we prove the following algebraic analogue to Theorem 4.1.

THEOREM 5.4. A homomorphism $\varphi^{\prime}: \pi_{1}(X) \rightarrow B(n)$ represents the trivial $n$-fold covering space over $X$ if and only if there exists a homomorphism $\varphi^{\prime \prime}: \pi_{1}(X) \rightarrow H(n)$ such that $\varphi^{\prime}=\rho_{n} \circ \varphi^{\prime \prime}$.

Proof. $\varphi^{\prime}: \pi_{1}(X) \rightarrow B(n)$ represents the trivial $n$-fold covering space over $X$ if and only if $\phi=\tau_{n} \circ \varphi^{\prime}$ is conjugate to, and therefore equal to, the constant homomorphism of $\pi_{1}(X)$ into the identity permutation in $\Sigma_{n}$. By exactness of the braid group sequence, it follows easily, that this is the case if and only if there exists a homomorphism $\phi^{\prime \prime}: \pi_{1}(X) \rightarrow H(n)$ such that $\varphi^{\prime}=\rho_{n} \circ \varphi^{\prime \prime}$.

ExAmPLE 5.5. Suppose that $\pi_{1}(X)$ is a torsion group. Since $B(n)$ by a theorem of Fox and Neuwirth [3] contains no torsion, the constant homomorphism into the identity element is the only homomorphism from $\pi_{1}(X)$ into $B(n)$. Since this homomorphism lifts to the constant homomorphism of $\pi_{1}(X)$ into $H(n)$, it follows 
therefore by Theorem 5.4, that every polynomial covering space over $X$ is trivial. This gives a slightly different proof of Theorem 4.3 in [6].

Finally, we turn now to the problem when two homomorphisms $\varphi, \psi \in \operatorname{Hom}\left(\pi_{1}(X), B(n)\right)$ determine the same equivalence class of $n$-fold polynomial covering spaces over $X$.

By the classification theorem for covering spaces, we know that two homomorphisms $\varphi, \psi \in \operatorname{Hom}\left(\pi_{1}(X), B(n)\right)$ determine the same equivalence class of $n$-fold polynomial covering spaces over $X$ if and only if $\tau_{n} \circ \varphi, \tau_{n} \circ \psi \in \operatorname{Hom}\left(\pi_{1}(X), \Sigma_{n}\right)$ are conjugate homomorphisms. Since $\tau_{n}$ is an epimorphism, this condition is satisfied if and only if there exists an element $b \in B(n)$ such that $\tau_{n} \circ \psi=\tau_{n} \circ \varphi^{b}$. Here $\varphi^{b} \in \operatorname{Hom}\left(\pi_{1}(X), B(n)\right)$ denotes the conjugation of $\varphi$ by $b$, i.e., for every $\gamma \in \pi_{1}(X)$ we have $\phi^{b}(\gamma)=b \cdot \varphi(\gamma) \cdot b^{-1}$. If $\tau_{n} \circ \psi=\tau_{n} \circ \varphi^{b}$, then exactness of the braid group sequence shows that there exists a unique map (not necessarily a homomorphism) $\theta: \pi_{1}(X) \rightarrow H(n)$ such that $\left(\rho_{n} \circ \theta\right) \cdot \psi=\varphi^{b}$. The multiplication on the left in this equation is pointwise multiplication of maps of $\pi_{1}(X)$ into $B(n)$.

Since $\left(\rho_{n} \circ \theta\right) \cdot \psi$ has to be a homomorphism of $\pi_{1}(X)$ into $B(n)$, $\theta$ has to satisfy a certain condition. To describe this condition, we note that the homomorphism $\psi: \pi_{1}(X) \rightarrow B(n)$ induces an action of $\pi_{1}(X)$ on $H(n)$ as follows. For $\gamma \in \pi_{1}(X)$ and $c \in H(n)$ we let $c^{\psi(\gamma)} \in$ $H(n)$ denote the unique element, which exists by exactness of the braid group sequence, such that

$$
\rho_{n}\left(c^{\psi(\gamma)}\right)=\psi(\gamma) \cdot \rho_{n}(c) \cdot \psi(\gamma)^{-1} \cdot
$$

The condition, the map $\theta: \pi_{1}(X) \rightarrow H(n)$ has to satisfy in order that $\left(\rho_{n} \circ \theta\right) \cdot \psi$ is a homomorphism of $\pi_{1}(X)$ into $B(n)$, is then easily seen to be expressed in the following

DeFINITION 5.6. Let $\psi: \pi_{1}(X) \rightarrow B(n)$ be a homomorphism. map $\theta: \pi_{1}(X) \rightarrow H(n)$ is then called a $\psi$-crossed homomorphism if

$$
\theta\left(\gamma_{1} \cdot \gamma_{2}\right)=\theta\left(\gamma_{1}\right) \cdot \theta\left(\gamma_{2}\right)^{\psi\left(\gamma_{1}\right)}
$$

for all $\gamma_{1}, \gamma_{2} \in \pi_{1}(X)$.

Using the notation above, the arguments preceeding Definition 5.6 proves the following

THEOREM 5.7. Two homomorphisms $\varphi, \psi \in \operatorname{Hom}\left(\pi_{1}(X), B(n)\right)$ determine the same equivalence class of $n$-fold polynomial covering spaces over $X$ if and only if there exists an element $b \in B(n)$ and $a$ 
$\psi$-crossed homomorphism $\theta: \pi_{1}(X) \rightarrow H(n)$ such that $\left(\rho_{n} \circ \theta\right) \cdot \psi=\varphi^{b}$.

Theorem 5.1 and Theorem 5.7 taken together provide a satisfactory algebraic description of the equivalence classes of polynomial covering spaces over $X$. It would be nice to have a geometric description, say in terms of a classifying space for the $n$-fold polynomial covering spaces over $X$.

\section{REFERENCES}

1. V. I. Arnol'd, On some topological invariants of algebraic functions, Trudy Moskov. Mat. Obshch., 21 (1970), 27-46. = Trans. Moscow Math. Soc., 21 (1970), 30-52.

2. E. Fadell and L. Neuwirth, Configuration spaces, Math. Scand., 10 (1962), 111-118.

3. R. Fox and L. Neuwirth, The Braid groups, Math. Scand., 10 (1962), 119-126.

4. E. A. Gorin and V. Ja. Lin, Algebraic equations with continuous coefficients, and certain questions of the algebraic theory of braids, Mat. Sb., 78 (120) (1969), 579-610. = Math. USSR Sbornik, 7 (1969), 569-596.

5. — On separable polynomials over commutative Banach algebras, Dokl. Akad. Nauk SSSR, 218 (1974), 505-508. = Soviet Math. Dokl., 15 (1974), 1357-1361.

6. V. L. Hansen, Coverings defined by Weierstrass polynomials, J. Reine Angew. Math., (to appear).

7. E. H. Spanier, Algebraic Topology, McGraw-Hill Book Company, 1966.

8. N. E. Steenrod, The Topology of Fibre Bundles, Princeton Mathematical Series, Princeton University Press, Princeton, N.J., 1951.

9. Ju. V. Zjuzin and V. Ja. Lin, Unramified algebraic extensions of commutative Banach algebras, Mat. Sb., 91 (133) (1973), 402-420. = Math. USSR Sbornik, 20 (1973), 419-437.

Received June 29, 1978.

KøBENHAVNS UNIVERSITET

MATEMATISK INSTITUT

KøBENHAVN, DANMARK 


\title{
PACIFIC JOURNAL OF MATHEMATICS
}

\section{EDITORS}

\author{
DONALD BABBITT (Managing Editor) \\ University of California \\ Los Angeles, CA 90024 \\ HUGo ROSSI \\ University of Utah \\ Salt Lake City, UT 84112 \\ C. C. MOORE \\ University of California \\ Berkeley, CA 94720
}

J. DugundJI

Department of Mathematics

University of Southern California

Los Angeles, CA 90007

R. FinN and J. Milgram

Stanford University

Stanford, CA 94305

\section{ASSOCIATE EDITORS}
E. F. BECKENBACH
B. H. Neumann
F. WolF
K. YOSHIDA

\section{SUPPORTING INSTITUTIONS}

\author{
UNIVERSITY OF BRITISH COLUMBIA \\ CALIFORNIA INSTITUTE OF TECHNOLOGY \\ UNIVERSITY OF CALIFORNIA \\ MONTANA STATE UNIVERSITY \\ UNIVERSITY OF NEVADA, RENO \\ NEW MEXICO STATE UNIVERSITY \\ OREGON STATE UNIVERSITY \\ UNIVERSITY OF OREGON
}

UNIVERSITY OF SOUTHERN CALIFORNIA

STANFORD UNIVERSITY

UNIVERSITY OF HAWAII

UNIVERSITY OF TOKYO

UNIVERSITY OF UTAH

WASHINGTON STATE UNIVERSITY

UNIVERSITY OF WASHINGTON

The Supporting Institutions listed above contribute to the cost of publication of this Journal, but they are not owners or publishers and have no responsibility for its content or policies.

Mathematical papers intended for publication in the Pacific Journal of Mathematics should be in typed form or offset-reproduced, (not dittoed), double spaced with large margins. Please do not use built up fractions in the text of the manuscript. However, you may use them in the displayed equations. Underline Greek letters in red, German in green, and script in blue. The first paragraph or two must be capable of being used separately as a synopsis of the entire paper. Please propose a heading for the odd numbered pages of less than 35 characters. Manuscripts, in triplicate, may be sent to any one of the editors. Please classify according to the scheme of Math. Reviews, Index to Vol. 39. Supply name and address of author to whom proofs should be sent. All other communications should be addressed to the managing editor, or Elaine Barth, University of California, Los Angeles, California, 90024.

50 reprints to each author are provided free for each article, only if page charges have been substantially paid. Additional copies may be obtained at cost in multiples of 50 .

The Pacific Journal of Mathematics is issued monthly as of January 1966. Regular subscription rate: $\$ 72.00$ a year (6 Vols., 12 issues). Special rate: $\$ 36.00$ a year to individual members of supporting institutions.

Subscriptions, orders for numbers issued in the last three calendar years, and changes of address should be sent to Pacific Journal of Mathematics, P.O. Box 969, Carmel Valley, CA 93924, U.S.A. Older back numbers obtainable from Kraus Periodicals Co., Route 100, Millwood, NY 10546.

PUBLISHED BY PACIFIC JOURNAL OF MATHEMATICS, A NON-PROFIT CORPORATION

Printed at Kokusai Bunken Insatsusha (International Academic Printing Co., Ltd.). 8-8, 3-chome, Takadanobaba, Shinjuku-ku, Tokyo 160, Japan.

Copyright (C) 1979 by Pacific Journal of Mathematics Manufactured and first issued in Japan 


\section{Pacific Journal of Mathematics}

\section{Vol. 81, No. $2 \quad$ December, 1979}

Ersan Akyildiz, Vector fields and equivariant bundles ............... 283

Ehrhard Behrends, The centralizer of tensor products of Banach spaces ( $a$ function space representation) ......................... 291

Geoffrey R. Burton, Congruent sections of a convex body ............. 303

John Warnock Carlson, $H$-closed and countably compact extensions ...... 317

Robert Charles Carlson, Eigenfunction expansions for selfadjoint

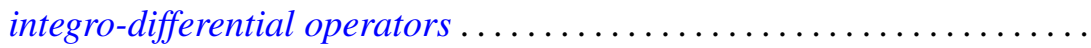

Robert Damiano, Coflat rings and modules.

Eric Karel van Douwen and Washek (Vaclav) Frantisek Pfeffer, Some properties of the Sorgenfrey line and related spaces ................

Uri Elias, Necessary conditions and sufficient conditions for disfocality and

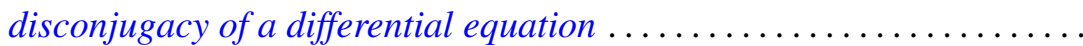

V. L. (Vagn Lundsgaard) Hansen, Polynomial covering spaces and homomorphisms into the braid groups .......................

Paul Hess, Dedekind's problem: monotone Boolean functions on the lattice

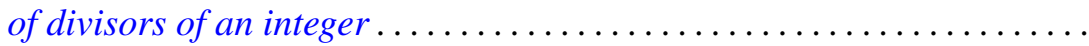

Alan Hopenwasser and David Royal Larson, The carrier space of a reflexive

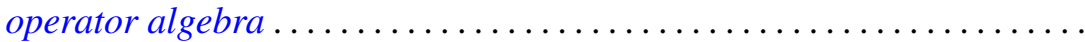

Kyung Bai Lee, Spaces in which compacta are uniformly regular $G_{\delta} \ldots \ldots$.

Claude Levesque, A class of fundamental units and some classes of

Jacobi-Perron algorithms in pure cubic fields...

Teck Cheong Lim, A constructive proof of the infinite version of the

Belluce-Kirk theorem ..........................

Dorothy Maharam and A. H. Stone, Borel boxes

Roger McCann, Asymptotically stable dynamical systems are linear

Peter A. McCoy, Approximation and harmonic continuation of axially symmetric potentials in $E^{3} \ldots \ldots \ldots \ldots \ldots \ldots \ldots$

Takahiko Nakazi, Extended weak-* Dirichlet algebras ....

Carl L. Prather, On the zeros of derivatives of balanced trigonometric polynomials ................................

Iain Raeburn, An implicit function theorem in Banach spaces...

Louis Jackson Ratliff, Jr., Two theorems on the prime divisors of zeros in completions of local domains...

Gloria Jean Tashjian, Cartesian-closed coreflective subcategories of

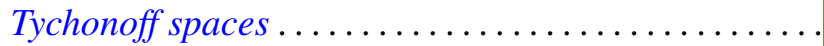

Stephen Edwin Wilson, Operators over regular maps.... . . 